\title{
A Physical Frequency-Dependent Compact Model for RF Integrated Inductors
}

\author{
Javier Sieiro, José M. López-Villegas, Member, IEEE, José Cabanillas, Student Member, IEEE, Joan A. Osorio, and \\ Josep Samitier, Associate Member, IEEE
}

\begin{abstract}
A frequency-dependent compact model for inductors in high ohmic substrates, which is based on an energy point-of-view, is developed. This approach enables the description of the most important coupling phenomena that take place inside the device. Magnetically induced losses are quite accurately calculated and coupling between electric and magnetic fields is given by means of a delay constant. The later coupling phenomenon provides a modified procedure for the computation of the fringing capacitance value, when the self-resonance frequency of the inductor is used as a fitting parameter. The model takes into account the width of every metal strip and the pitch between strips. This enables the description of optimized layout inductors. Data from experiments and electromagnetic simulators are presented to test the accuracy of the model.
\end{abstract}

Index Terms-Eddy currents, inductor layout optimization, inductor model, RF ICs, self-resonance.

\section{INTRODUCTION}

$\mathbf{O}$ NEOFTHE limiting factors in the development of RFintegrated circuit(IC) designs has been the absence of high-performance integrated passive components, mainly inductors and transformers. Low self-resonant frequency (SRF), due to the electrical coupling, and high losses in the substrate and in the metal strips deteriorate their characteristics. However, the current state of the art has improved their performance with the introduction of novel techniques compatible with standard IC industry.

Bulk [1] and surface micromachining [2] are two of the best solutions. With these techniques, the substrate underneath the component is removed and the substrate losses and electrical coupling through it are eliminated. The use of high resistivity substrates has also been demonstrated to be effective [3]. Metal losses can be reduced with the use of low-resistivity metals, such as copper [3]. In addition, the incorporation of ground shields in the design [4] reduce the losses in the substrate, but only those associated to the electrical coupling.

Once the substrate losses are small, magnetically induced losses in the metal regions become important. In order to solve this problem, advances in the modeling of inductors and transformers have also led up to a new kind of technique based on the optimization of the layout [5]-[7] and on the differential excitation of the inductor [8], [9]. In the first case, the designer tries to find the optimum value between the magnetic induced losses and ohmic losses in the metal strips. It is worth stressing

Manuscript received May 1, 2001. This work was supported by the Spanish Science and Technology Commission under Project TIC98-0836-C02-01 and Project TIC98-0836-C02-02.

The authors are with the Department of Electronics, University of Barcelona, E-08028 Barcelona, Spain.

Publisher Item Identifier S 0018-9480(02)00849-9. that no technological effort is required in the implementation of this technique and that performance can be excellent, as demonstrated in [5]. Regarding the second case, it is possible to minimize the voltage between the strips and, consequently, to shift up the SRF of the component.

In spite of all technological efforts, there is still a major drawback from the designer's perspective: the lack of accurate scalable compact models. This is a serious problem because the effects of parasitics in passive devices can have a dramatic impact on the performance of the circuit. In order to solve this problem, electromagnetic simulators can be used to predict the behavior of passive devices with a high degree of accuracy. Nevertheless, the designer must have some expertise with electromagnetic solvers and a longer design time is required due to the increase in computation time. Moreover, there is no information about the behavior of the device versus changes in geometry, which could be provided by scalable analytical equations. Therefore, the use of compact models for the description of the inductors and other components is required to speed up the analysis phase of the design. Notice that not only the analysis but the synthesis phase benefits from the use of compact models, since it is possible to identify the relevant key points for the development of optimization algorithms.

There are several approaches to the modeling of inductors that can be found in the literature [10]-[26]. Simple compact models predict the inductance value at the dc behavior using closed formulas or algorithmic methods. More complex compact models are able to describe the behavior of the component up to the first SRF. However, all of them compute the different lumped elements using a static field analysis. In this work, we introduce new concepts in the development of the compact model. The main characteristics of the model are the introduction of all layout dimensions, the accurate computation of the eddy currents in the metal strips, the introduction of the effect of a ground plane in the inductance value, and a first approach to the analysis of propagation phenomena.

This paper is organized as follows. In Section II, a survey on compact modeling techniques and frequency phenomena is presented. The compact model is developed in Section III. The objective is to obtain a scalable physical lumped model accounting for important frequency phenomena, which are usually neglected. The accuracy of this model is demonstrated in Section IV. Finally, conclusions are given in Section V.

\section{Physical Phenomena IN Inductors}

Fig. 1 represents a typical lumped electrical compact model for inductors. From an energy point-of-view, the different el- 


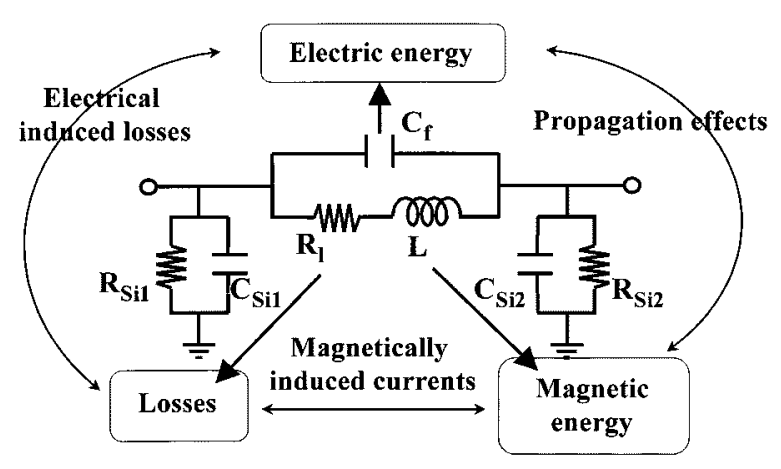

Fig. 1. Ideal compact model for RF IC inductors.

ements can be associated with physical phenomena. The ideal inductor describes the total amount of magnetic energy; the capacitors account for the electrical coupling between metal strips and ground plane; and the resistors collect the losses in metal and substrate. However, all these three energy quantities (magnetic and electric energy and losses) are not independent from each other and the coupling between them must be taken into account. The interaction between electric and magnetic fields will induce a propagation phenomenon and, therefore, the device will present a set of self-resonant frequencies. Aside from the ohmic losses, the magnetic and electric fields will also induce losses on the conductive regions. Accordingly, if all previously mentioned contributions are dismissed, the model will not predict properly the frequency behavior. In the literature, many models try to develop this frequency approach. However, the main problem is that these models deal with the electric and magnetic energy of the static fields.

Excluding the case of numerical techniques such as the method of moments or the partial elements equivalent circuit (PEEC), most of the models compute the inductance value in the dc behavior. Closed expressions based on a fitting geometrical procedure can be found in [15]. These expressions are very helpful as a first guess of the inductance value over a wide range of typical inductor geometries. Other authors have reported closed forms based on physical assumptions [11], but the accuracy is lower due to the multivariable nature of the problem. Algorithmic techniques seem to be the best procedure to increase the accuracy of the computation. One of the most widespread methods is due to Greenhouse [12], and it is based on the work of Grover [10]. The key point of the technique is the calculation of the mutual inductance between two strips using the geometric mean distance (GMD). However, the GMD concept is an approximation of the Neumann integral and its accuracy is plotted in Fig. 2. The trace shows an increasing error when the length of the strip is equal or shorter than its width. To fulfill this gap, other works have developed more accurate expressions [13], [14]. Such a kind of algorithmic procedure is also followed in this paper, but two additional concepts are added. Firstly, the energy associated between pairs of strips is evaluated, rather than their mutual magnetic interaction. As we will show, this allows the introduction of propagation phenomena. Secondly, the expressions are evaluated for different widths of the metal strips. This evaluation is very important when one tries to increase the quality factor $(Q)$ of the inductor by layout optimization. If we take into account

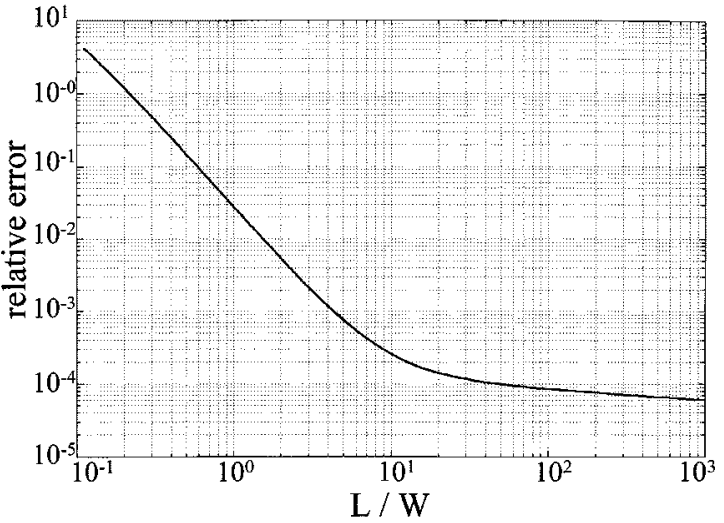

Fig. 2. Relative error on the metal strip self-inductance value calculated with the GMD approach as a function of the length/width ratio.

the width of each metal strip and the pitch between strips, the optimization space is expanded and significant improvement on the $Q$ value can be achieved [5].

In spite of being important, magnetically induced losses in the substrate and in the metal strips (eddy currents) are not considered in most of the compact models. Their description requires a frequency-dependent resistor and the development of simple expressions is not an easy task. Fitting expressions for particular cases can be found in the literature [14]. Recently, several simple expressions have been developed for the losses in the metal, based on the skin depth value [17]. One can also find more accurate expressions based on a first-order calculation of the electric and magnetic coupling in the metal regions [18], [19]. In this paper, new formulas are explored to explain the losses in the metal.

A frequency-dependent value for the inductance is expected due to the distribution of the eddy currents. In the case of high resistivity substrates, this variation is small. However, for CMOS substrates, the eddy currents drop significantly the value of the inductance [20]. This fact has either not been taken into account in compact models or has been modeled with a simple resistor value. In our approach, the model is devoted to high resistivity substrates, so it will not be the subject of further discussion.

The SRF gives the frequency limit application of a given inductor. The frequency limit value is related to the coupling of the electric and magnetic fields, so the wavelength has a value of a typical dimension of the inductor. Within the compact model, the main purpose of the capacitors is to take into account all the electrical couplings to describe the SRF. The capacitance matrix is a suitable procedure to compute the energy associate to the electrical couplings. However, due to the multilayered nature of ICs, the Green's function of the media becomes very complicated and numerical methods must be used [21], [22]. Within the compact modeling framework, the capacitance matrix is usually substituted by simple parallel capacitance formula [17] for the evaluation of the underpass bridge and the coupling to the substrate. With these approximations, the accuracy of the SRF value is low. Thus, in such a case, the capacitor values are left as fitting parameters to experimental or electromagnetic simulation data for a good agreement. The usual fitting procedure is based on the SRF formula

$$
\mathrm{SRF}=\frac{1}{2 \pi \sqrt{L C}} \sqrt{1-\frac{R^{2} C}{L}}
$$


where $L$ and $R$ are related to the static magnetic field and the ohmic losses. Here, an alternative fitting procedure related to the wave propagation phenomena inside the inductor is presented.

\section{EleCtrical SCALABLE Model}

Normally, inductors for RF IC applications must have equivalent inductance values in the range of few nanohenrys, SRFs in the range of several gigahertz, and high values of the quality factor. Due to the degrading effects of the substrate (losses and capacitive coupling), all these requirements are difficult to fulfill. In the case of bipolar technologies, the magnetically induced losses in the substrate are less severe and Fig. 1 is a suitable lumped element model. The magnetic energy is represented with $L$, the ohmic losses and the magnetically induced losses are described by the $R_{l}$ series resistor, and the electrical coupling between the strips is taken into account with the $C_{f}$ capacitor. Finally, the substrate effects are described with $C_{S i 1,2}$ (the electrical coupling to the substrate) and $R_{S i 1,2}$ (the losses in the substrate). Notice that silicon micromachining inductors can also be modeled with this scheme. Hereafter, these parameters are going to be related to the physics of the inductor (the following discussion is an extension of a previous work [23]).

\section{A. Inductor}

In microelectronic technologies, the typical shapes for planar inductors are the rectangular and circular spirals. One way to compute their inductance value is through the well-known relationship between the energy stored in the component and the current flowing through it as follows:

$$
E=\frac{1}{2} L|I|^{2} .
$$

The energy can be calculated through the integration of the term $\mathbf{A} \cdot \mathbf{J}^{*}$ over the volume defined by the conductor, where $\mathbf{A}$ is the magnetic vector potential and $\mathbf{J}$ is the current distribution. Of course, using electromagnetic simulators, this can be accomplished for any shape of the metal strips (e.g., circular and rectangular spirals).

In order to obtain analytical expressions, symmetry arguments are very useful. In the case of circular spirals, there is still some axial symmetry. Thus, it is possible to approximate the geometry to a model based on concentric loops. This last step can be done using two different criteria: 1) the perimeter of each circular loop must equal the perimeter of each turn of the spiral or 2) the area of each loop must be the same as the average area of each spiral turn. Choosing between the two criteria depends, basically, on the pitch value. For instance, for small pitches, the first criterion would give a better description, while for large values the second one is better. Based on these assumptions, fast numerical techniques can be developed by means of a series expansion of the Green's function [24].

In the case of rectangular spiral inductors, the division of the geometry according to each metal strip is a suitable way for having an accurate computation of the inductance value. In fact, this is the procedure followed by most accurate compact models [14], [17]. The difference among them lies in the way they compute the mutual coupling of the strips.
Once the geometry has been divided in a simple way, the magnetic energy can be obtained by partitioning the integral $\int_{V} \boldsymbol{A} \cdot \boldsymbol{J}^{*} d v$ in each one of the metal strip volumes and by splitting the contribution to the vector potential in terms of their density current distribution, shown as

$$
E=\frac{1}{2} \sum_{i, j} \int_{v_{j}} A_{i} \cdot J_{j}^{*} d v=\frac{1}{2} \sum_{i, j} E_{i j} .
$$

The term $E_{i j}$ is the energy associated to each pair of loops (circular topology) or each pair of metal strips (rectangular topology). Now, the problem has been reduced to the calculation of the term $E_{i j}$. In the case of the circular spirals, the integral form of $E_{i j}$ is

$$
E_{i j}=\frac{\mu_{o}}{2} \int_{a_{j}}^{b_{j}} r J_{j}(r)\left(\int_{a_{i}}^{b_{i}} \int_{0}^{2 \pi} \frac{r^{\prime} J_{i}\left(r^{\prime}\right) \cos \varphi^{\prime}}{\left|r-r^{\prime}\right|} d r^{\prime} d \varphi^{\prime}\right) d r
$$

where $\mu_{o}$ is the permeability of the free space, $a_{i}$ and $a_{j}$ are the inner radii of the loop $\mathbf{i}$ and $\mathbf{j}$, respectively, $b_{i}$ and $b_{j}$ are the outer radii, and $J_{i}(r)$ and $J_{j}(r)$ are the current distributions. Whereas Eddy currents in the metal strips are not important, the density current distribution for the circular loop is very close to the dc distribution and is given by

$$
J(r)=\frac{I}{\ln \left(\frac{b}{a}\right) r} .
$$

Introducing (4) into (3) and using the generating function of the Legendre's polynomials, the former integral can be solved analytically. The solution is given by

$$
E_{i j}=\mu \frac{I_{i}}{\ln \left(b_{i} / a_{i}\right)} \frac{I_{j}^{*}}{\ln \left(b_{j} / a_{j}\right)} \sum_{n=0}^{\infty} C_{2 n+1} D_{2 n+1}
$$

where the coefficients $C_{2 n+1}$ are

$$
C_{2 n+1}=\frac{n}{n+1}\left(\frac{2 n-3}{n-1}-1\right) C_{n-2}, \quad C_{1}=1
$$

and the coefficients $D_{2 n+1}$ are a function of the current distribution in each loop. For a circular loop in the dc regime they are

$$
\begin{aligned}
& D_{2 n+1}^{i=j}=\frac{\left[(2 n+1) r+\frac{a_{i}^{2 n+2}}{r^{2 n+1}}\right]_{a_{i}}^{b_{i}}+\left[(2 n+2) r+\frac{r^{2 n+2}}{b_{i}^{2 n+1}}\right]_{a_{i}}^{b_{i}}}{(2 n+2)(2 n+1)} \\
& D_{2 n+1}^{i \neq j}=\frac{\left[r^{-(2 n+1)}\right]_{b_{j}}^{a_{j}}\left[r^{(2 n+2)}\right]_{a_{i}}^{b_{i}}}{(2 n+2)(2 n+1)}
\end{aligned}
$$

Other expressions for this problem, based on elliptic integrals, can be found in [14]. The accuracy of (6) is supported by results from magnetic simulations. These simulations have been performed with ANSYS 5.6.2 quasi-static solver (based on the method of finite elements), and with MoMemtum solver (based on the method of moments) for a family of inductors having a width of $20 \mu \mathrm{m}$, a pitch of $40 \mu \mathrm{m}$, and an inner radius of $120 \mu \mathrm{m}$. Table I shows the inductance values obtained using both simulators and (6). 
TABLE I

INDUCTANCE VALUE FOR CIRCULAR INDUCTORS

\begin{tabular}{c|c|c|c}
\hline Turns & ANSYS & MoM & Eq.(6) \\
\hline 1 & $0.53 \mathrm{nH}$ & $0.53 \mathrm{nH}$ & $0.53 \mathrm{nH}$ \\
\hline 2 & $1.81 \mathrm{nH}$ & $1.81 \mathrm{nH}$ & $1.81 \mathrm{nH}$ \\
\hline 3 & $3.96 \mathrm{nH}$ & $3.97 \mathrm{nH}$ & $3.96 \mathrm{nH}$ \\
\hline 4 & $6.99 \mathrm{nH}$ & $6.87 \mathrm{nH}$ & $7.09 \mathrm{nH}$ \\
\hline
\end{tabular}

The former procedure can be applied to square inductors. In this case, the term $E_{i j}$ takes into account the magnetic energy due to the coupling between the strips $i$ and $j$. The approximation of an infinitely thin sheet current is now applied. Notice that only the coupling between parallel strips contributes to the energy value. Locating the center of one of the metal strips at the origin, the $E_{i j}$ integral to solve is

$$
\begin{aligned}
E_{i j}=\frac{\mu_{o} I_{i} I_{j}^{*}}{32 \pi W_{i} W_{j}} \int_{x_{i}-L_{i}}^{x_{i}+L_{i}} \int_{y_{i}-W_{i}}^{y_{i}+W_{i}} \int_{-L_{j}}^{L_{j}} \int_{-W_{j}}^{W_{j}} & \frac{d x_{i} d y_{i} d x_{j} d y_{j}}{\sqrt{\left(x_{i}-x_{j}\right)^{2}+\left(y_{i}-y_{j}\right)^{2}}}
\end{aligned}
$$

where $2 L_{i}, 2 L_{j}$ are the lengths of the metal strips, $2 W_{i}$ and $2 W_{j}$ are their widths, and $\left(x_{i}, y_{i}\right)$ is the center of the second metal strip. The solution of integral (9) is

$$
\begin{aligned}
E_{i j}= & \frac{\mu_{o} I_{i} I_{j}^{*}}{32 \pi W_{i} W_{j}}\left[\left[\left[[F(x, y)]_{y+W_{i}}^{y-W_{i}}\right]_{y_{j}-W_{j}}^{y_{j}+W_{j}}\right]_{x+L_{i}}^{x-L_{i}}\right]_{x_{j}+L_{j}}^{x_{j}+L_{j}} \\
F(x, y)= & x y^{2} \operatorname{sh}^{-1}\left(\frac{x}{|y|}\right)+y x^{2} \operatorname{sh}^{-1}\left(\frac{y}{|x|}\right) \\
& -\frac{1}{3}\left(x^{2}+y^{2}\right)^{3 / 2} .
\end{aligned}
$$

The superindexes and the subindexes in the right brackets read as the limits of integration. Thus, using the Barrow's rule for definite integrals and starting from the inner bracket, the complete expression is developed. Similar expressions can be found elsewhere [13], [14] with the same degree of accuracy.

Equation (10) has also been tested with the electromagnetic simulator MoMemtum, and with the Greenhouse method. The results are shown in Table II, where the dimensions of the coils used are a strip width of $100 \mu \mathrm{m}$, a pitch of $200 \mu \mathrm{m}$, and an inner side of $900 \mu \mathrm{m}$ just to be compared with reference [12]. Notice that differences between the exact calculation of the term $E_{i j}$ and by using the GMD concept are actually small due to the inherent geometry of the inductor.

The model for the inductance value presented in this section has two main advantages. Firstly, it fully enables the description of the geometry in a full sense because takes into account the width of each metal strip. To illustrate this point, Fig. 3 shows the layout of an optimized inductor [5], where a different metal strip width for each turn has been obtained. For the low-frequency range, the measured value of the inductor is $32.25 \mathrm{nH}$. Currently available compact models are not able to predict the magnetic behavior for this kind of structures. With the model presented in this subsection, the computed inductance value is
TABLE II

INDUCTANCE VALUE FOR SOUARE INDUCTORS

\begin{tabular}{c|c|c|c}
\hline Turns & MoM & Ref [12] & Eq.(10) \\
\hline 1 & $2.25 \mathrm{nH}$ & $2.74 \mathrm{nH}$ & $2.74 \mathrm{nH}$ \\
\hline 2 & $9.27 \mathrm{nH}$ & $9.31 \mathrm{nH}$ & $9.32 \mathrm{nH}$ \\
\hline 3 & $20.24 \mathrm{nH}$ & $20.44 \mathrm{nH}$ & $20.44 \mathrm{nH}$ \\
\hline 4 & $36.88 \mathrm{nH}$ & $37.02 \mathrm{nH}$ & $37.03 \mathrm{nH}$ \\
\hline
\end{tabular}

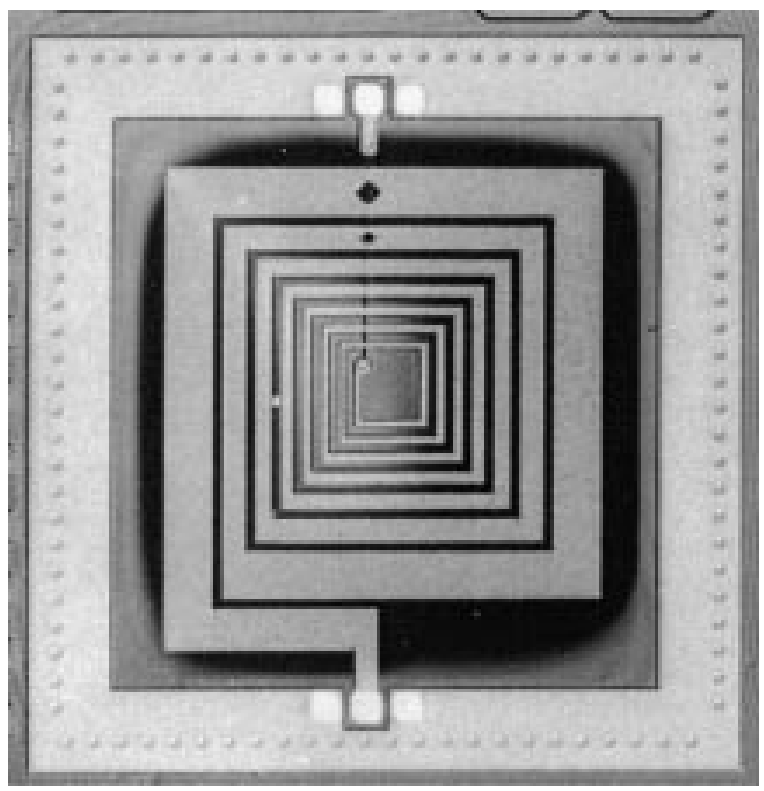

Fig. 3. Layout of an optimized inductor.

$34.69 \mathrm{nH}$. Note that the difference between both values is a consequence of the mirrored coil due to the ground plane. The introduction of a new term in the Green's function of the vector potential will overcome this problem. Ideally, this term represents the image of the current distribution of the coil through the ground plane. The vector potential integral form becomes

$$
A(r)=\int_{V} \frac{J\left(r^{\prime}\right)-J_{\text {image }}\left(r^{\prime}\right)}{\left|r^{\prime}-r\right|} d v
$$

and the computed inductance value is now $32.62 \mathrm{nH}$.

The second advantage comes directly from the energy point-of-view adopted in the calculation. Notice that, in (6) and (10), the currents through each metal strip are kept explicitly. It suggests that any delay between the currents in the different metal strips will change the magnetic stored energy in the inductor and, therefore, the actual inductance value. Moreover, the maximum stored energy occurs when all the currents are in phase, i.e., in the dc regime, where no propagation exists. Thus, due to the propagation of the electromagnetic fields, this energy is a decreasing function of the frequency, at least until the SRF of the inductor is reached. In the following subsection, the importance of this phenomenon is explored, when a fitting procedure is used to extract the fringing capacitance $C_{f}$.

\section{B. Capacitors}

Compact model capacitors account for the electrical coupling between both ports and ground. Many efforts have been directed 
to calculate their values, but numerical techniques are required to compute this coupling, due to the multilayered structure of ICs [21]. Some authors use microstrip transmission line theory to account for the interactions between neighbor metal strips [16], [25]. Also, other simple models based on parallel capacitance formula can be found [17], [26].

If radiation terms are neglected, Poisson's equation describes the electric field inside the structure [20]. Equivalently, it can be described in terms of the capacitance matrix $C_{i j}$ of the inductor. In such case, the electrical energy $E_{e l}$ is given by

$$
E_{e l}=\frac{1}{2} \sum_{i, j}^{N, N} C_{i j} V_{i} V_{j}
$$

The $i$ and $j$ indexes run over the metal strips, $V_{i}$ and $V_{j}$ are the voltage values, and $N$ are the number of strips.

The simplest way to relate the capacitance matrix with the compact model capacitors is to assume a linear voltage drop along the length of the inductor

$$
V_{i}=V_{1}+\frac{V_{2}-V_{1}}{l} x_{i}
$$

where $V_{i}$ is the voltage of the strip $i, V_{1}$ and $V_{2}$ are the voltages at the ports, $l$ is the total length of the inductor, and $x_{i}$ is the length coordinate related to the strip $i$. With that assumption, $C_{s i 1}, C_{s i 2}$, and $C_{f}$ are given by

$$
\begin{aligned}
\frac{\partial^{2} E_{e l}}{\partial V_{1}^{2}} & =C_{s i 1} \\
\frac{\partial^{2} E_{e l}}{\partial V_{2}^{2}} & =C_{s i 2} \\
\frac{\partial E_{e l}}{\partial V_{1} \partial V_{2}} & =C_{f} .
\end{aligned}
$$

Except in the case of very simple substrates, this technique requires lengthy calculations. For more complicated substrates, other simple methods are preferred. An interesting option is presented in [17], where the three capacitances are calculated with simple closed expressions. However, if one needs a good description near the SRF, it is better to evaluate $C_{s i 1}$ and $C_{s i 2}$ as a parallel capacitance and leave $C_{f}$ as a fitting parameter. In fact, $C_{f}$ includes most of the technology information (thickness and dielectric constant of all layers).

However, a direct fitting procedure using (1) contradicts some physical facts. For example, when increasing the length of the inductor, the value of the capacitor should also increase. Fig. 4 shows the value of the fitted capacitance as a function of the number of strips in the inductor (see [5] for a description of the substrate). Surprisingly, the capacitance decreases in certain zones; therefore, $C_{f}$ has not a clear meaning. Notice that the values on the plot where the capacitance increases are due to the underpass bridge.

Hereafter, a modified fitting procedure based on an ac analysis of the inductance is outlined. Let us suppose that there is a phase shift between currents in the turns. For that reason, the value of the inductance close to the SRF will be lower than that at the dc regime. Therefore, if the dc value of the inductance is

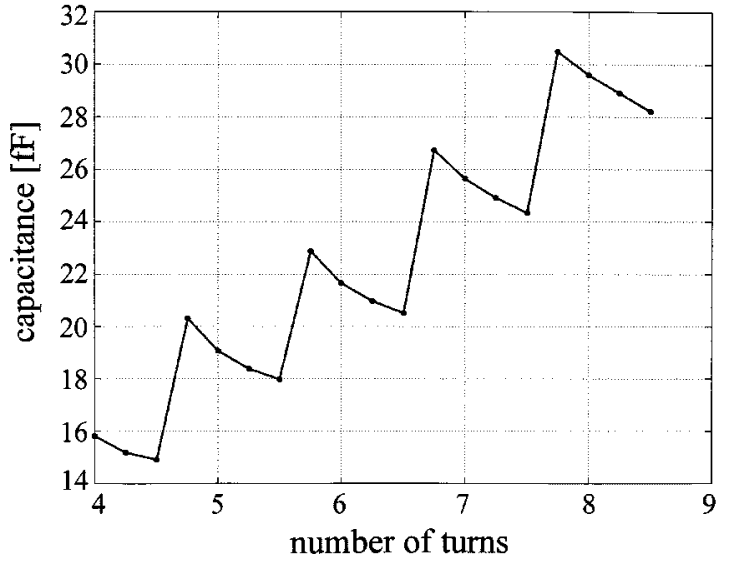

Fig. 4. $C_{f}$ capacitance value computed as a fitting parameter to the SRF versus the number of turns. Geometry of the inductors: width $w=20 \mu \mathrm{m}$, pitch $p=40 \mu \mathrm{m}$, and inner side length $d_{i}=95 \mu \mathrm{m}$.

used in (1), the fitting capacitor will then underestimate the actual value. In such a situation, the equivalent inductance will be also different for frequencies below the self-resonance.

Nevertheless, the electrical coupling is also expected to increase with frequency due to the increase of the odd mode propagation in the structure. Thus, the actual capacitance value is a function of frequency and can hide the former phenomena. In order to check that, one can assume a frequency dependence of the inductor $L$ and the capacitor $C_{f}$. A first-order expansion in frequency can then be developed as follows:

$$
\begin{gathered}
L(f)=L_{\mathrm{DC}}-\frac{\Delta L}{\Delta f} f \\
C_{f}(f)=C_{f}(0)+\frac{\Delta C_{f}}{\Delta f} f .
\end{gathered}
$$

The equivalent inductance value of the component is now defined as

$$
L_{\mathrm{eq}}=\frac{L}{1-L C(2 \pi f)^{2}} .
$$

Then, substituting (15a) into (16) and keeping the value of $C$ constant, the ratio between the actual $L_{\mathrm{eq}}$ and the equivalent inductance of the compact model, $L_{\text {eq_ideal }}$, is

$$
\frac{L_{\mathrm{eq}}}{L_{\text {eq_ideal }}} \approx 1-\frac{\frac{\Delta L}{\Delta f}}{L_{\mathrm{DC}}} f .
$$

Repeating the same procedure with (15b), but keeping $L$ constant, the perturbation introduced by the term $\Delta C_{f} / \Delta_{f}$ is given by $(17 b)$

$$
\frac{L_{\mathrm{eq}}}{L_{\text {eq_ideal }}} \approx 1+\theta\left(f^{2}\right)
$$

where $\theta\left(f^{2}\right)$ is a second-order frequency term. Therefore, (17) reflects that the actual equivalent inductance value will be lower than the prediction made by an ideal lumped compact model.

In order to check the behavior of (17), an inductor with no loss (except that related to the radiation) is studied with the aid of an electromagnetic simulator. In this case, an interesting quantity to plot is the ratio between both equivalent inductances $L_{\mathrm{eq}}$ 


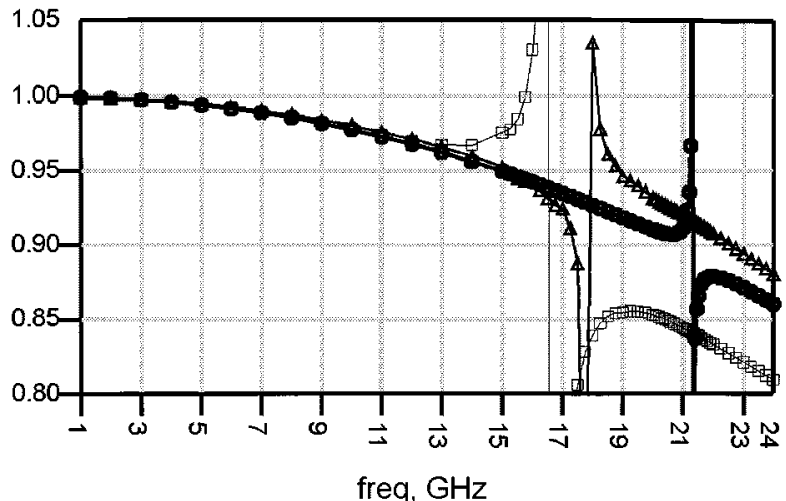

Fig. 5. $L_{\text {cq }} / L_{\text {cq-iccal }}$ ratio for three inductors: $(\square) p=50 \mu \mathrm{m}, d_{i}=90 \mu \mathrm{m}$, $N=5, w=30 \mu \mathrm{m} ;(\Delta) p=50 \mu \mathrm{m}, d_{i}=90 \mu \mathrm{m}, N=5, w=20 \mu \mathrm{m}$; (O) $p=50 \mu \mathrm{m}, d_{i}=90 \mu \mathrm{m}, N=4, w=20 \mu \mathrm{m}$.

and $L_{\text {eq-ideal }}$ because it is a direct measured of the actual difference between $L_{\mathrm{DC}}$ and $L(f)$, see (15a). This result is shown in Fig. 5. There is an increasing difference between both values when the frequency is increasing. As a consequence, (1) should be replaced by

$$
\mathrm{SRF}=\frac{1}{2 \pi \sqrt{L(f) C}} \sqrt{1-\frac{R(f)^{2} C}{L(f)}} .
$$

The main problem is now how to introduce a frequency-dependent inductor $L(f)$, so that (18) can be computed. In fact, the solution has already been given in (10). Let us assume the existence of a phase delay among the strip currents due to the propagation of the electromagnetic waves inside the structure. Now, $I_{i}$ and $I_{j}$ are phasors and, in a first-order approximation, they are given by

$$
I_{i}=|I| e^{-j \beta x_{i}}
$$

where $\beta$ is a propagation phase constant. Notice that $\beta$ depends weakly on the geometry, as shown in Fig. 5. The three different inductors have the same behavior until their SRF. Substituting (19) into (10) and equating it to an actual simulated or measured $L$ value close to the SRF, the value of $\beta$ is found for a wide range of geometries. If a better accuracy is required, one can perform the simulation or the measurement of a few inductors covering the geometry range of interest. Once $\beta$ is known, the inductance at any frequency below the SRF is computed with the algorithm explained in Section III-A. Fig. 6 shows the full agreement between data calculated using the analytical formulation and results obtained using from the electromagnetic simulator. Thus, if a fitting procedure is used to find $C_{f}$, one can conclude that the modification introduced by (19), instead of the $L_{\mathrm{DC}}$ value, is a better approximation for the value of $L(f)$ in (18).

\section{Resistors}

Regarding the substrates studied in this paper, losses inside the substrate are mainly due to the capacitive coupling. They are described with $R_{S i 1}$ and $R_{S i 2}$. These two resistors are easily calculated just by adding an imaginary part to the dielectric constant that accounts for the substrate conductance. Two different

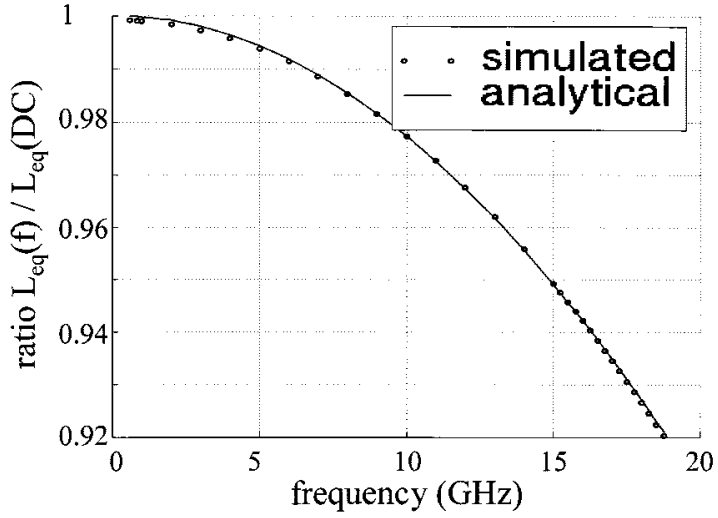

Fig. 6. Estimate of the phase delay effect on the inductance value.
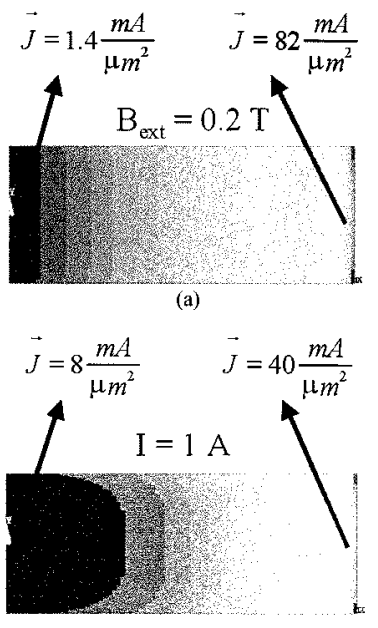

(b)

Fig. 7. Current density distribution in a metal strip: (a) due to an external magnetic field and (b) due to a feeding current. By symmetry, only half of the width is modeled.

approaches to find their value are given in [17] and [26], thus no further development is done in this paper.

Ohmic losses, due to the conduction current, and magnetically induced losses (eddy currents) are also important. In fact, they determine the quality factor of the inductor. Their contribution is included inside the compact model with the resistor $R_{l}$. Ohmic losses are directly related to the sheet resistance of the metal strip and the geometry of the inductor. Losses due to eddy currents can be evaluated once the redistribution of the current in the inductor is known. Although skin effect has been proposed as a solution to compute the value of $R_{l}$, it is not consistent with the expected physical behavior. For the metal strip geometry used in the design of inductors, electromagnetic simulations show that the distribution of the current takes place along the width of the strip, and not across its thickness. This result can be seen in Fig. 7 for two cases: 1) there is a transversal magnetic field and 2) a current is feeding the strip. A good approximation to compute the current density distribution can be found in [18] and [19]. A more precise analysis is next developed.

Our starting point is the integral equation for the current density distribution $\boldsymbol{J}$ in terms of the Green's function of the vector 
potential $\boldsymbol{A}$. If the metal is an ohmic material, $\boldsymbol{J}$ can be written as follows:

$$
\boldsymbol{J}=\sigma \boldsymbol{E}+\boldsymbol{J}_{\text {src }}
$$

where $\sigma$ is the conductivity of the metal, $\boldsymbol{E}$ is the induced electric field inside the metal region, and $\boldsymbol{J}_{\boldsymbol{s r c}}$ is the impressed current. Then, the eddy current distribution $\boldsymbol{J}_{\text {eddy }}$ is given by

$$
\begin{array}{r}
J_{\text {eddy }}(\boldsymbol{r})=-j \omega \sigma\left[\int_{V} G^{K}\left(\boldsymbol{r}, \boldsymbol{r}^{\prime}\right)\left(\boldsymbol{J}_{\text {eddy }}\left(\boldsymbol{r}^{\prime}\right)+\boldsymbol{J}_{\text {src }}\left(\boldsymbol{r}^{\prime}\right)\right) d v^{\prime}\right. \\
\left.+\boldsymbol{A}_{\text {ext }}(\boldsymbol{r})\right]
\end{array}
$$

where $G^{K}\left(r, r^{\prime}\right)$ is the Green's function of the vector potential, and $A_{\text {ext }}$ is an external vector potential. From this equation, one must realize about the existence of two eddy current sources. The first source is the impressed current $\boldsymbol{J}_{s r c}$ in the volume, and the second is the external field $\boldsymbol{A}_{\text {ext }}$. In the case of an inductor, the external magnetic field in a metal strip is due to the magnetic coupling with all the remaining strips. In order to simplify the calculation, one can assume that $A_{\text {ext }}$ is not affected by the current redistribution, i.e.,

$$
\begin{aligned}
A(r)= & \int_{V i} G^{K}\left(\boldsymbol{r}, \boldsymbol{r}^{\prime}\right) \boldsymbol{J}_{i}\left(\boldsymbol{r}^{\prime}\right) d v^{\prime} \\
& +\sum_{j \neq i}^{N} \int_{V j} G^{K}\left(\boldsymbol{r}, \boldsymbol{r}^{\prime}\right) \boldsymbol{J}_{j}\left(\boldsymbol{r}^{\prime}\right) d v^{\prime} \\
\approx & \int_{V i} G^{K}\left(\boldsymbol{r}, \boldsymbol{r}^{\prime}\right) \boldsymbol{J}_{i}\left(\boldsymbol{r}^{\prime}\right) d v^{\prime} \\
& +\sum_{j \neq i}^{N} \int_{V j} G^{K}\left(\boldsymbol{r}, \boldsymbol{r}^{\prime}\right) \boldsymbol{J}_{s c r_{-j}}\left(\boldsymbol{r}^{\prime}\right) d v^{\prime}
\end{aligned}
$$

where $\boldsymbol{J}_{\boldsymbol{i}}, \boldsymbol{J}_{j}$ are the density current distribution at the metal strips $i$ and $j$, and $J_{s r c_{-} j}$ is the impressed current in strip $j$. Therefore, (21) becomes

$$
\begin{array}{r}
\boldsymbol{J}_{\text {eddy }}(\boldsymbol{r}) \\
=-j \omega \sigma\left[\int_{V i} G^{K}\left(\boldsymbol{r}, \boldsymbol{r}^{\prime}\right)\left(J_{e d d y_{-} i}\left(\boldsymbol{r}^{\prime}\right)+\boldsymbol{J}_{s r c_{-} i}\left(\boldsymbol{r}^{\prime}\right)\right) d v^{\prime}\right. \\
\left.+\sum_{j \neq i}^{N} \int_{V j} G^{K}\left(\boldsymbol{r}, \boldsymbol{r}^{\prime}\right) \boldsymbol{J}_{s c_{-j} j}\left(\boldsymbol{r}^{\prime}\right) d v^{\prime}\right] \cdot
\end{array}
$$

The external field is now directly related to the impressed current and a relation between $\boldsymbol{J}_{s r c}$ and $\boldsymbol{J}_{\text {eddy }}$ can be found. The integral equation (23) does not have a closed analytical solution and numerical methods must be used to solve the problem.

It is possible to simplify the problem based on a simple mesh of the strip, where no end effects are considered. In this case, the relevant physics of the problem is completely taken into account. Fig. 8 shows the mesh and conditions to analyze the eddy currents due to an external field. From symmetry considerations, two opposite sign currents will flow through the edges of the metal strip. Recalling (22), the external magnetic field from the remaining metal strips can be written in a simple way as

$$
\boldsymbol{B}_{\text {ext }}=\nabla \times \boldsymbol{A} \Rightarrow \boldsymbol{B}_{\text {ext }}(\boldsymbol{r})=G(\boldsymbol{r}) I
$$

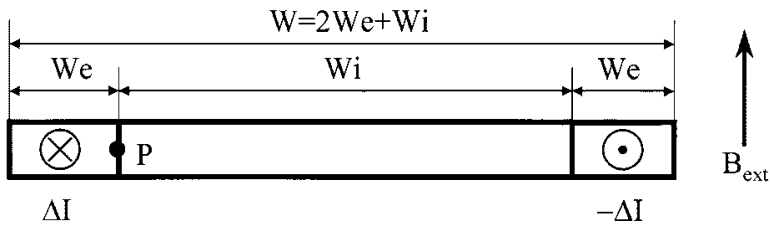

Fig. 8. Mesh geometry for the computation of the magnetically induced currents in a metal strip.

where $G(r)$ is a function of the inductor geometry, and $I$ is the impressed current. The total magnetic field at the point $r=$ $P$, including the effect of the unknown eddy currents, can be expressed then with the equation

$$
B(P)=G(P) I-\frac{4 \mu_{o} \Delta I}{\pi W} \frac{1+q}{(1-q)(1+3 q)}
$$

where $\mu_{o}$ is the permeability of free space, $W$ is the width of the strip, $q$ is the ratio of $W_{i} / W$ ( $W_{i}$ is the width of the mesh at the center of the strip), and $\Delta I$ is the unknown magnetically induced current. Notice that $\Delta I$ is, in general, a complex number.

From Faraday's law, the induced electric field is

$$
E=j W \pi f \frac{1-q}{4}\left(G(P) I-\frac{4 \mu_{o} \Delta I}{\pi W} \frac{1+q}{(1-q)(1+3 q)}\right)
$$

Finally, using Ohm's law, the unknown $\Delta I$ can be solved in a closed expression as a function of $I$

$$
\Delta I=\frac{K \Lambda+j \Lambda}{1+K^{2}} I .
$$

$K$ and $\Lambda$ are defined as follows:

$$
\begin{aligned}
K & =\frac{\mu_{o} W f\left(1-q^{2}\right)}{\left.2 R_{s}(1+3 q)\right)} \\
\Lambda & =\frac{(1-q)^{2} W^{2} \pi G f}{8 R_{s}}
\end{aligned}
$$

where $R_{s}$ is the sheet resistance of the metal. The main difference with any other previously developed expression is that the phase between the eddy current and the impressed current appears naturally. The reason is that the redistribution of the magnetic field due to the eddy currents has also been included in the derivation. Now, the losses and the contribution, namely, $R_{l_{-} \text {ext }}$, to the resistor value $R_{l}$ can easily be calculated as

$$
\begin{aligned}
P_{\text {loss }} & =\sum_{i=1}^{3} R_{s_{i}}\left|I_{i}\right|^{2} \\
& =2 R_{s}|\Delta I|^{2} \\
& =\frac{2 R_{\mathrm{DC}}}{q \lambda} \frac{\left(\frac{f}{f_{o}}\right)^{2}}{1+\left(\frac{f}{f_{o}}\right)^{2}}|I|^{2} \\
R_{l_{-} \text {ext }} & =\frac{P_{\text {loss }}}{|I|^{2}} .
\end{aligned}
$$




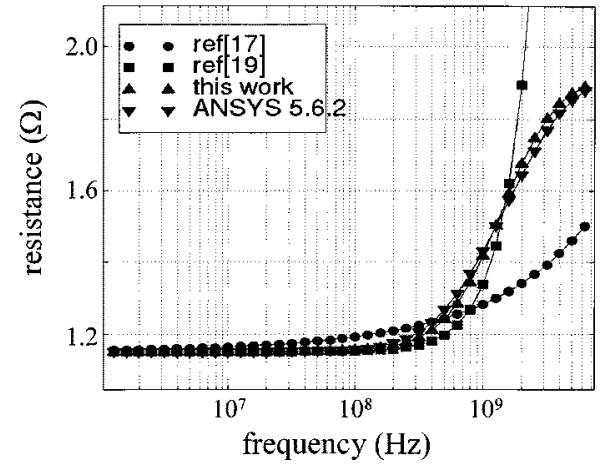

Fig. 9. Frequency behavior of the resistor value $R_{l}$. Inductor geometry: $w=$ $20 \mu \mathrm{m}, p=40 \mu \mathrm{m}, d_{i}=60 \mu \mathrm{m}, N=4$, and $R_{s}=20 \mathrm{~mW}$.

The values of $f_{o}$ and $\lambda$ are

$$
\begin{aligned}
\lambda & =\left(\frac{4 \mu_{o}(1+q)}{\pi W G(1+3 q)}\right)^{2} \\
f_{o} & =\frac{2 R_{s}(1+3 q)}{\mu_{o} W\left(1-q^{2}\right)} .
\end{aligned}
$$

The same procedure can be applied for the computation of the eddy currents due to an impressed current in the metal strip. In that case, the final solution for the contribution to the resistance value is given by

$$
P_{\mathrm{loss}}=R_{\mathrm{DC}}\left(1+\frac{4 q}{(1-q)^{2}} \frac{\left(\frac{f}{f_{o}}\right)^{2}}{1+\left(\frac{f}{f_{o}}\right)^{2}}\right)|I|^{2} .
$$

Finally, the value of $R_{l}$ is found by the addition of the two contributions to the losses, i.e., (29a) and (31),

$$
R_{l}=R_{\mathrm{DC}}+R_{\mathrm{RF}} \frac{\left(\frac{f}{f_{o}}\right)^{2}}{\left(1+\left(\frac{f}{f_{o}}\right)^{2}\right)} .
$$

In (32), $R_{\mathrm{DC}}$ accounts for the ohmic losses, $R_{\mathrm{RF}}$ is related to the magnetically induced losses due to the external magnetic field and due to the current through the conductor at very high frequencies, and $f_{o}$ is a frequency factor that controls the transition from the low-frequency to the high-frequency behavior. We would like to stress the fact that all the parameters in (32) are not fitting parameters, but values obtained from analytical expressions.

Once more, electromagnetic simulators can be used to demonstrate the predicted behavior. Fig. 9 shows the comparison between the losses in an inductor computed with a quasi-static numerical simulator, and the results given by (32) and [17] and [19]. In [19] an approximate behavior is given until the frequency $f_{o}$ is reached; but then, it diverges from the actual value because the phase of the induced current has not been properly computed.
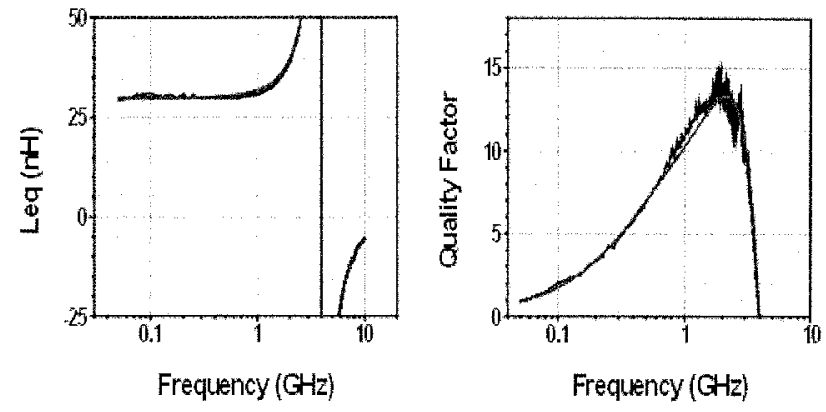

Fig. 10. Comparison between developed model and experimental results. Inductor geometry: $w=51 \mu \mathrm{m}, p=75 \mu \mathrm{m}, d_{i}=110 \mu \mathrm{m}$, and $N=7$ ).

\section{EXPERIMENTAL RESULTS}

The accuracy of the developed compact model is compared directly with the experimental data of a micromachined inductor. We must stress the fact that this model has only one fitting parameter, i.e., the capacitance $C_{f}$, obtained with the modified fitting procedure of Section III-B. The results are given in Fig. 10, where a very good agreement is shown until some frequency range after the SRF of the inductor is reached.

\section{CONCLUSION}

This paper has presented relevant results of an exhaustive analysis of integrated inductors. The main and new features of the developed model are the ability to evaluate the equivalent inductance, including the phase shift in the current, and the frequency-dependent series resistance by means of analytical expressions derived from physical considerations. In addition, this model introduces as a new set of variables the width of each metal strip, which enables the study and development of layout optimization algorithms. A modified method to compute the value of the fringing capacitance $C_{f}$ has also been given. The accuracy of the model has been proved with actual measurements.

It has also been shown that electromagnetic simulators are valuable tools in order to develop scalable models. With them, one can quantify the contribution of each particular physical phenomenon that takes place in the inductor. The physical-based frequency-dependent compact model developed in this paper can easily be implemented inside commercial design environments to obtain high-quality inductors in the design of oscillators, LNA, and other MMIC-RFIC circuits.

\section{ACKNOWLEDGMENT}

The authors thank Dr. B. Rejaei, ECTM/DIMES Group, Delft University of Technology, Delft, The Netherlands, for his valuable insights.

\section{REFERENCES}

[1] J. Y. C. Chang, A. A. Abidi, and M. Gaitan, "Large suspended inductors on silicon and their use in a $2-\mu \mathrm{m}$ CMOS RF amplifier," IEEE Electron Device Lett., vol. 14, pp. 246-258, May 1993.

[2] H. Jiang, Y. Wang, J. A. Yeh, and N. C. Tien, "On-chip spiral inductors suspended over deep copper-lined cavities," IEEE Trans. Microwave Theory Tech., vol. 48, pp. 2415-2423, Dec. 2000. 
[3] J. N. Burghartz, D. C. Edelstein, M. Soyuer, H. A. Ainspan, and K. A. Jenkins, "RF circuit aspects of spiral inductors on silicon," IEEE J. Solid-State Circuits, vol. 33, pp. 2028-2033, Dec. 1998.

[4] C. P. Yue and S. S. Wong, "On-chip spiral inductors with patterned ground shields for Si-based RFIC's," IEEE J. Solid-State Circuits, vol. 33, pp. 734-752, May 1998.

[5] J. M. López-Villegas, J. Samitier, C. Cané, and P. Losantos, "Improvement of the quality factor of RF integrated inductors by layout optimization," IEEE Trans. Microwave Theory Tech., vol. 48, pp. 76-83, Jan. 2000.

[6] J. Craninckx and M. S. J. Steyaert, "A 1.8-GHz low-phase-noise CMOS VCO using optimized hollow spiral inductors," IEEE J. Solid-State Circuits, vol. 32, pp. 736-744, May 1997.

[7] J. E. Post, "Optimizing the design of spiral inductors on silicon," IEEE Trans. Circuits Syst. II, vol. 47, pp. 15-17, Jan. 2000.

[8] M. Danesh, J. R. Long, R. A. Hadaway, and D. L. Harame, "A Q-factor enhancement technique for MMIC inductors," in IEEE MTT-S Int. Microwave Symp. Dig., vol. 1, 1998, pp. 183-186.

[9] H. B. Erzgräber, M. Pierschel, G. G. Fischer, Th. Grabola, and A. Wolf, "High performance integrated spiral inductors based on a minimum AC difference voltage principle," in Silicon Monolithic Integrated Circuits RF Syst. Topical Meeting Dig., 2000, pp. 71-74.

[10] F. W. Grover, Inductance Calculations, 2nd ed. New York: D. Van Nostrand, 1947.

[11] F. E. Terman, Radio Engineering Handbook. New York: McGrawHill, 1943, pp. 48-60.

[12] H. M. Greenhouse, "Design of planar rectangular microelectronic inductors," IEEE Trans. Parts, Hybrids, Packag., vol. PHP-10, pp. 101-109, June 1974.

[13] C. Hoer and C. Love, "Exact inductance equations for rectangular conductors with applications to more complicated geometries," J. Res. Natl. Bur. Stand. C, vol. 69, no. 2, pp. 127-137, Apr.-June 1965.

[14] E. Pettenpaul, H. Kapusta, A. Weisgerber, H. Mampe, J. Luginsland, and I. Wolff, "CAD models of lumped elements on GaAs up to $18 \mathrm{GHz}$," IEEE Trans. Microwave Theory Tech., vol. 36, pp. 294-304, Feb. 1988.

[15] S. S. Mohan, M. M. Hershenson, 89S. P. Boyd, and T. H. Lee, "Simple accurate expressions for planar spiral inductors," IEEE J. Solid-State Circuits, vol. 34, pp. 1419-1425, Oct. 1999.

[16] J. R. Long and M. A. Copeland, "The modeling, characterization, and design of monolithic inductors for silicon RF IC's," IEEE J. Solid-State Circuits, vol. 32, pp. 357-369, Mar. 1997.

[17] C. P. Yue and S. S. Wong, "Physical modeling of spiral inductors on silicon," IEEE Trans. Electron Devices, vol. 47, pp. 560-568, Mar. 2000.

[18] J. M. López-Villegas, J. Samitier, C. Cané, and P. Losantos, "Improvement of the quality factor of RF integrated inductors by layout optimization," in IEEE Radio Freq. Integrated Circuits Symp., 1998, pp. $169-172$.

[19] W. B. Kuhn and N. M. Ibrahim, "Analysis of current crowding effects in multiturn spiral inductors," "Physical modeling of spiral inductors on silicon," IEEE Trans. Microwave Theory Tech., vol. 49, pp. 31-38, Jan. 2001.

[20] A. M. Niknejad and R. G. Meyer, "Analysis of eddy-current losses over conductive substrates with applications to monolithic inductors and transformers," IEEE Trans. Microwave Theory Tech., vol. 49, pp. 166-176, Jan. 2001.

[21] A. Niknejad, R. Gharpurey, and R. G. Meyer, "Numerically stable Green function for modeling and analysis of substrate coupling in integrated circuits," IEEE Trans. Computer-Aided Design, vol. 17, pp. 305-315, Apr. 1998.

[22] Z. Jiang, P. S. Excell, and Z. M. Hejazi, "Calculation of distributed capacitances of spiral resonators," IEEE Trans. Microwave Theory Tech., vol. 45, pp. 139-142, Jan. 1997.

[23] J. Sieiro, J. M. López-Villegas, J. Cabanillas, J. A. Osorio, and J. Samitier, "A complete physical frequency dependent lumped model for RF integrated inductors," in IEEE Radio Freq. Integrated Circuits Symp., 2001, pp. 121-124.

[24] B. Rejaei, D. C. van der Pol, and J. L. Tauritz, "Analysis of circular spiral inductors on CMOS substrates," in Eur. Solid-State Device Res. Conf., 1999, pp. 672-675.

[25] K. C. Gupta, R. Garg, and I. Bahl, Microstriplines and Slotlines, 2nd ed. Norwood, MA: Artech House, 1996

[26] Y. K. Koutsoyannopoulos and Y. Papananos, "Systematic analysis and modeling of integrated inductors and transformers in RFIC design," IEEE Trans. Circuits Syst. II, vol. 47, pp. 699-713, Aug. 2000.

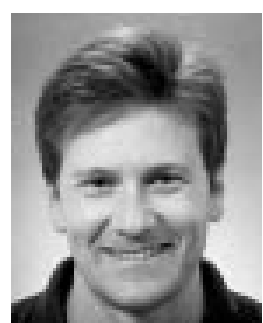

Javier Sieiro was born in Barcelona, Spain, in 1971. $\mathrm{He}$ received the Physics degree and M.S. degree in electronic engineering from the University of Barcelona, Barcelona, Spain, in 1995 and 1999, respectively, and is currently working toward the $\mathrm{Ph} . \mathrm{D}$. degree in electronics at the University of Barcelona.

His current research areas are the modeling of passive components for RFIC applications and the design of $L C$ voltage-controlled oscillators.

José M. López-Villegas (M'94) was born in Barcelona, Spain, in 1962. He received the Physics degree and Ph.D. degree in physics from the University of Barcelona, Barcelona, Spain, in 1985 and 1990, respectively.

From 1985 to 1987, he was a Visiting Research Fellow at the Laboratoire d'Electronic Philips (LEP), Paris, France, where he was involved with the electrical characterization of III-V compound semiconductor devices. In 1990, he joined the Departament of Electronics, University of Barcelona, initially as an Assistant Professor and then as an Associate Professor. He currently manages projects dealing with the design and test of RFICs performed using silicon and microsystems technologies.

José Cabanillas (S'01) was born in Palma de Mallorca, Balearic Islands, Spain, in 1973. He received the M.S. degree in electronic engineering from the University of Barcelona, Barcelona, Spain, in 1998, and is currently working toward the Ph.D. degree in electronics at the University of Barcelona

His current research areas are the modeling of transformers for RFIC applications and the design of $L C$ voltage-controlled oscillators and mixers.

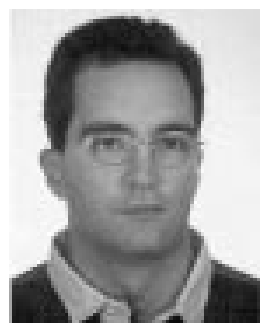

Joan A. Osorio was born in Escaldes-Engordany, Andorra, Spain, in 1975. He received the B.S. degree in telecommunications engineering and the M.S. degree in electronic engineering from the University of Ramon Llull, Barcelona, Spain, in 1997 and 1999 , respectively, and is currently working toward the Ph.D. degree in electronics at the University of Barcelona, Barcelona, Spain.

His research concerns the development of small and compact antennas for industrial-scientific-medical (ISM) bands.

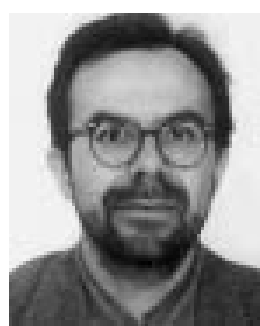

Josep Samitier (M'95-A'95) received the Ph.D. degree from the University of Barcelona, Barcelona, Spain, in 1986.

He is currently a Full Professor of electronic instrumentation with the Electronic Department, University of Barcelona, and Director of the Instrumentation and Communication Systems (SIC) research group since February 1995. In March 2001, he became the Director of the Electronic Department and Deputy Head of the Scientific and Technological Park, University of Barcelona. From 1977 to 1982, he studied physics at the University of Barcelona. From 1983 to 1984, he was a Research Fellow in the Applied Physics Department, University of Barcelona, in the field of GaAs MESFET devices and electrooptical characterization of III-V semiconductors. From February 1984 to June 1985, he was a Visiting Research Fellow at the Laboratoire d'Electronique Philips (LEP), Paris, France. In 1988, he became an Assistant Professor of electronics at the same university, involved in the development of microsystems devices and electronic. He has also been a consultant for automotive industries in the field of sensors and distributed instrumentation and control systems development. Over the last ten years, he has participated in several European projects concerning integrated microsystems based on silicon technology. His current research and developed projects concern the design, modeling, and test of microsystems and the design of interface circuits for signal conditioning of sensors and actuators. 